\title{
Dietary patterns in urban Ghana and risk of type 2 diabetes
}

\author{
Laura K. Frank ${ }^{1}$, Janine Kröger ${ }^{1}$, Matthias B. Schulze ${ }^{1}$, George Bedu-Addo ${ }^{2}$, Frank P. Mockenhaupt ${ }^{3}$ \\ and Ina Danquah ${ }^{1 *}$ \\ ${ }^{1}$ Department of Molecular Epidemiology, German Institute of Human Nutrition Potsdam-Rebbruecke, \\ Arthur-Scheunert-Allee 114-116, Nuthetal 14588, Germany \\ ${ }^{2}$ Komfo Anokye Teaching Hospital, School of Medical Sciences, Kwame Nkrumah University of Science and Technology, \\ University Post Office, Kumasi, Ghana \\ ${ }^{3}$ Institute of Tropical Medicine and International Health, Charité - Universitäetsmedizin, Spandauer Damm 130, \\ Berlin 14050, Germany
}

(Submitted 10 June 2013 - Final revision received 17 December 2013 - Accepted 31 January 2014 - First published online 8 April 2014)

\section{Abstract}

There is epidemiological evidence for associations between dietary patterns and type 2 diabetes. However, for sub-Saharan Africa, information on dietary patterns and their contribution to diabetes is lacking. The aim of the present study was to identify dietary patterns and their associations with type 2 diabetes in an urban Ghanaian population. In a hospital-based case-control study on risk factors for type 2 diabetes in Kumasi, a FFQ was administered to 675 controls and 542 cases. Dietary patterns were identified by using factor analysis including thirty-three food items. Logistic regression was used to evaluate the associations of dietary patterns with type 2 diabetes. Overall, two dietary patterns were identified: (1) a 'purchase' dietary pattern which positively correlated with the consumption of sweets, rice, meat, fruits and vegetables and (2) a 'traditional' dietary pattern that correlated with the intake of fruits, plantain, green leafy vegetables, fish, fermented maize products and palm oil. In the highest quintile of the 'purchase' dietary pattern, participants were younger, leaner and of higher socio-economic status than those in the lower quintiles. In contrast, participants in the highest quintile of the 'traditional' dietary pattern were older, heavier and more deprived compared with those in the lower quintiles. In the multivariate model, the 'purchase' dietary pattern was inversely associated with type 2 diabetes (OR per 1 SD $0.41,95 \%$ CI 0.33, 0.50); the 'traditional' dietary pattern increased the odds of diabetes per 1 sD by $54 \%$ (95\% CI 1.35, 1.81). In conclusion, two diverse dietary patterns were identified and associated with type 2 diabetes in urban Ghana. The determinants of pattern adherence require further investigation.

Key words: Dietary patterns: Type 2 diabetes: Nutrition: Factor analysis: Sub-Saharan Africa

Clearly, nutritional behaviour is one of the major modifiable risk factors for type 2 diabetes. In nutritional epidemiology, the principal approach in the past was to investigate the association between single nutrients or foods and a disease. However, the human diet is very complex: foods are not consumed independently and nutrients act synergistically. This justifies the consideration of overall dietary patterns. Generally, dietary patterns are constructed by either an a priori or an $a$ posteriori approach ${ }^{(1-4)}$. The a priori method takes into account nutrition recommendations that are evidence-based for nutrition-disease relationships. One popular and wellestablished dietary pattern from Caucasian populations is the Healthy Eating Index, based on the US Department of Agriculture Food Guide Pyramid ${ }^{(5)}$. Further indices have been proposed to reflect total dietary quality, such as the Dietary Quality Index based on the US dietary recommendations ${ }^{(6)}$.
The Dietary Quality Index takes into account nutrient adequacy, dietary variety, proportionality of food groups, and moderation of less healthy foods. With regard to a posteriori techniques, these are hypothesis-free, exploratory analyses based on the covariance structure of food variables. Either some sort of factor analysis is applied to identify underlying factors of food variables or cluster analysis is used to group individuals based on their similarities in food consumption data $^{(7)}$. Alternatively, mixed forms of dietary pattern construction exist that consider the pathway from diet to disease. For instance, the reduced-rank regression method identifies patterns of food consumption related to intermediate biomarkers. This method ensures the extraction of linear combinations of predictor variables $(=$ dietary factors) that explain as much response variation ( $=$ intermediate biomarker) as possible ${ }^{(8)}$.

Abbreviations: 24HDR, 24 h dietary recall; SES, socio-economic status; SSA, sub-Saharan Africa.

*Corresponding author: Dr I. Danquah, fax +4933200 88 2437, email ina.danquah@dife.de 
Numerous studies have identified prevailing dietary patterns in US, European and Asian populations, and investigated their association with the risk of diabetes ${ }^{(9-14)}$. Although the identified dietary patterns were somewhat populationspecific, there were notable similarities between the studies. Most of these studies have found a healthy pattern, characterised by a high consumption of healthy foods such as fruits, vegetables, fish, poultry and whole grains, which was associated with a reduced risk of type 2 diabetes, and a less healthy pattern, characterised by a high consumption of foods such as processed and red meats, fried foods, sweets and desserts, and refined grains, which was related to a higher risk of type 2 diabetes.

However, compared with other regions, food availability and consumption in sub-Saharan Africa (SSA) may be substantially different due to distinctions in climate, agriculture, food production and processing, and cultural habits.

Opposing the potential importance of nutritional behaviour for the emergence of type 2 diabetes in SSA, dietary patterns have only insufficiently been evaluated in this region. Therefore, the aim of the present study was to identify prevailing dietary patterns by using factor analysis in an urban Ghanaian population, and to evaluate their associations with type 2 diabetes.

\section{Experimental methods}

\section{Study population}

Between August 2007 and June 2008, an unmatched casecontrol study to identify risk factors for type 2 diabetes (and hypertension) was performed at the Komfo Anokye Teaching Hospital in Kumasi, Ghana. In this region, the prevalence rates of diabetes and overweight are $6 \%^{(15)}$ and $23 \%{ }^{(16)}$, respectively. A detailed description of the recruitment procedures and the characteristics of the study participants has been provided elsewhere ${ }^{(17,18)}$. In brief, cases were recruited from the diabetes centre $(n$ 495) and the hypertension clinic ( $n$ 451). They advertised the participation as potential controls among their friends, neighbours and community members ( $n$ 222). Similarly, further preliminary controls were recruited from the outpatient department ( $n$ 150) and among hospital staff ( $n$ 148). Each participant provided a fasting blood sample (fluoride plasma, tubes cooled at $+4^{\circ} \mathrm{C}$ ). Type 2 diabetic cases were defined as having fasting plasma glucose concentrations $\geq 7 \mathrm{mmol} / \mathrm{l}$ (Glucose $201^{+}$; HemoCue) and/or documented anti-diabetic medication ${ }^{(19)}$. Controls were defined as participants without diabetes. Of the 1466 participants included in the present study, 245 (16.7\%) were excluded from the present analysis due to missing information on nutrition (FFQ, 124 (8.5\%); $24 \mathrm{~h}$ dietary recall (24HDR), $17(1 \cdot 2 \%))$, anthropometry (39 (2.7\%)), socio-economic status (SES, $31(2 \cdot 1 \%)$ ) and genetic polymorphisms (34 (2.3\%)). Hence, this analysis comprised 1221 individuals (679 controls and 542 cases with diabetes).

The present study was conducted according to the guidelines laid down in the Declaration of Helsinki, and all procedures involving human patients were approved by the
Ethics Committee, School of Medical Sciences, University of Science and Technology, Kumasi. Written informed consent was obtained from all participants.

\section{Dietary assessments}

For nutrition assessment, a locally specific FFQ was designed. In face-to-face interviews, trained nurses speaking the local language applied the FFQ to all participants in a separate air-conditioned room after breakfast. The FFQ queried for the usual weekly consumption of fifty-one food items in ten food categories over the past 12 months: 'During the past 12 months, how often did you usually consume the following foods per week?' Food categories of the FFQ were based on the latest Ghana Demographic and Health Survey ${ }^{(20)}$. These categories were starchy roots and plantain; cereals and cereal products; animal products; legumes, nuts and oilseeds; fruits; vegetables; fats and oils; salt and spices; sweets; and liquids (see online supplementary Table S1). No portion sizes were available. Thus, the FFQ covered frequencies, but not quantities, of food consumption. There were six response categories: never; seldom ( $<1 \times$ per week); $1-2 \times$ per week; 3-4 $\times$ per week; $5-6 \times$ per week; daily. This FFQ has not been validated yet.

To describe energy and macronutrient intakes at the study population level, a single $24 \mathrm{HDR}$ was administered to each participant. Trained study personnel speaking the local language applied the 5-steps multiple-pass $\operatorname{method}^{(21)}$ in face-to-face interviews. Food and beverage consumption between midnight of the pre-last day and midnight of the last day was recorded in detail. Time and occasion of the meals were included. All consumed foods, their mode of preparation and their portion sizes, estimated by Ghanaian household utensils, were documented.

\section{Assessment of covariates}

As described previously ${ }^{(17)}$, all participants underwent a routine clinical examination. Weight $(\mathrm{kg})$, height $(\mathrm{cm})$, waist $(\mathrm{cm})$ and hip $(\mathrm{cm})$ circumferences were measured (all devices; SECA). BMI was calculated as weight $/(\text { height })^{2}\left(\mathrm{~kg} / \mathrm{m}^{2}\right)$ and the waist:hip ratio as waist circumference/hip circumference $^{(18)}$. Sociodemographic data and medical history were documented in face-to-face interviews by trained study personnel speaking the local language. These comprised age, sex, education (none, primary, secondary, tertiary or other) and occupation (subsistence farmer, commercial farmer, casual labourer, artisan, trader, businessman/woman, public servant, unemployed or other), own and family history of diabetes (yes or no) and smoking behaviour (never, quit or current). In these interviews, the duration (min/week) and type (i.e. intensity) of work-related, transportation-related and leisure-time physical activity were recorded. These data were translated into daily energy expenditure $(\mathrm{kJ} / \mathrm{d})$ as the sum of metabolic equivalents corresponding to activity intensity $(\mathrm{ml} / \mathrm{kg}$ per $\mathrm{min}) \times$ body weight $(\mathrm{kg}) \times$ duration $(\mathrm{min})^{(22)}$. 


\section{Statistical analyses}

Baseline characteristics of the study population were compared using non-parametric procedures, i.e. Mann-Whitney $U$ test for metric variables and $\chi^{2}$ test for categorical variables.

To describe nutritional behaviour, we started with exploring the usual nutrient consumption at the study population level. The daily intakes of energy $(\mathrm{kJ} / \mathrm{d})$, protein, carbohydrates, total fat and dietary fibre were determined $(\mathrm{g} / \mathrm{d})$ from the 24HDR. We converted the estimated portion sizes into grams ${ }^{(23)}$, and Ghana-specific and international nutrient composition tables were used to translate food consumption into nutrient intakes ${ }^{(24)}$. For comparisons of macronutrient intakes between the study groups, the respective values were standardised per $4184 \mathrm{~kJ} / \mathrm{d}(1000 \mathrm{kcal} / \mathrm{d})$.

The primary aim of the present analysis was to identify dietary patterns in this study population. Therefore, we used thirty-three food items or food groups from the FFQ for dietary pattern analysis. Of the fifty-one original food items of the FFQ, we collapsed and excluded some food items as described in online supplementary Table S1.

To identify underlying dietary patterns, factor analysis was performed using the PROC FACTOR procedure in SAS. The original food items were collapsed into latent factors explaining the maximum of the total variance of these thirty-three food-item variables. An orthogonal rotation (Varimax) was applied to ensure that the factors remained uncorrelated and to improve interpretability. To detect the optimal number of factors to be extracted, we used the criteria of an eigenvalue $>1$, the scree plot and plausibility of the factors. The factor score for each pattern was calculated by summing the intakes of standardised food intake (mean $=0$ and $S D=1$ ) weighted by their factor loadings. Each participant received a factor score for each identified dietary pattern. These scores were used to rank participants according to the degree to which they conformed to each dietary pattern. Factors were initially extracted separately for men and women. In a two-factor solution, the resulting rotated factor loadings of highly loading food items (rotated factor loadings >0.35) were similar between men and women (data not shown). Also, we calculated dietary patterns separately in the control group and in the total study population, and these results were merely identical (data not shown). Hence, we present the patterns derived among the complete study population.

Quintiles of dietary pattern scores were constructed based on the distribution among the control group. Baseline characteristics and frequencies of food intake were calculated across the quintiles of each dietary pattern score among the control group. The differences among categorical variables ( $\chi^{2}$ test) and linear trends among continuous parameters (trend tests) were compared.

Logistic regression analysis was applied to evaluate the associations between dietary patterns and type 2 diabetes. OR and 95\% CI were calculated across the quintiles and per $1 \mathrm{SD}$ of the factor score. The significance of a linear trend across the categories was tested by assigning each participant the median of a category and by modelling this value as a continuous variable. In our analyses, three different models were constructed: (1) model 1 was adjusted for age and sex; (2) model 2 included age, sex, family history of diabetes (yes or no), unemployment (yes or no), educational attainment (any or none), literacy (able or unable), smoking (current smoker, ex-smoker or never) and daily energy expenditure (kcal/d) as covariates; (3) model 3 included all variables of model 2 plus BMI and waist:hip ratio. All analyses were performed using SAS statistical software (version 9.2; SAS Institute).

\section{Results}

\section{Study population}

Table 1 illustrates that the study population was mainly female (76\%), middle-aged (mean $50 \cdot 4$ years) and of low SES (unemployment, 25\%; illiteracy, 35\%; lack of formal education, $25 \%)$. The participants with diabetes were significantly older (55 v. 47 years; $P<0.0001$ ), exhibited a higher waist:hip ratio (0.91 v. 0.86; $P<0.0001)$, had more often diabetes in the family (59 v. 25\%; $P<0.0001$ ) and tended to smoke more often than the controls ( $8 v .4 \% ; P=0.05)$. The SES in the diabetic group was lower than that in the control group, as observed by higher prevalence rates of educational alienation (17v. 35\%), illiteracy (26v. 46\%) and unemployment (16v $35 \%$; all $P<0 \cdot 0001)$. At the same time, energy expenditure was higher (Table 1).

\section{Nutritional behaviour}

Almost one-quarter of the study population consumed two meals per d (controls, 25\%; cases, 14\%; P<0.001), and $76 \%$ took three daily meals (controls, $73 \%$; cases, $84 \%$; $P<0.001$ ).

Table 1. Sociodemographic and anthropometric characteristics of 1221 urban Ghanaians

(Mean values and standard deviations; number of participants and percentages)

\begin{tabular}{|c|c|c|c|c|}
\hline \multirow[b]{2}{*}{ Characteristics } & \multicolumn{2}{|c|}{$\begin{array}{l}\text { Control } \\
(n 679)\end{array}$} & \multicolumn{2}{|c|}{$\begin{array}{c}\text { Diabetes } \\
\text { cases }(n 542)\end{array}$} \\
\hline & Mean & SD & Mean & SD \\
\hline Age (years) & $46 \cdot 8$ & $15 \cdot 8$ & $54.8^{*}$ & $13 \cdot 4$ \\
\hline $\mathrm{BMI}\left(\mathrm{kg} / \mathrm{m}^{2}\right)$ & $25 \cdot 8$ & $5 \cdot 4$ & $25 \cdot 8$ & $5 \cdot 1$ \\
\hline Waist:hip ratio & 0.86 & 0.08 & $0.91^{*}$ & 0.07 \\
\hline \multicolumn{5}{|l|}{ Energy expenditure } \\
\hline Median & \multirow{2}{*}{\multicolumn{2}{|c|}{$\begin{array}{c}1250 \\
915-1682\end{array}$}} & \multirow{2}{*}{\multicolumn{2}{|c|}{$\begin{array}{c}1526^{\star} \\
1039-2082\end{array}$}} \\
\hline IQR & & & & \\
\hline \multicolumn{5}{|l|}{$\mathrm{kJ} / \mathrm{d}$} \\
\hline \multirow{3}{*}{$\begin{array}{l}\text { Median } \\
\text { IQR }\end{array}$} & \multicolumn{2}{|c|}{5230} & \multirow{2}{*}{\multicolumn{2}{|c|}{$\begin{array}{c}6385 \\
4347-8711\end{array}$}} \\
\hline & 3828 & 7037 & & \\
\hline & $n$ & $\%$ & $n$ & $\%$ \\
\hline Sex (female) & 523 & $77 \cdot 0$ & 399 & $73 \cdot 6$ \\
\hline Family history of diabetes (positive) & 171 & $25 \cdot 2$ & $317^{*}$ & $58 \cdot 5$ \\
\hline Smoking (ever) & 29 & 4.3 & $41^{*}$ & 7.6 \\
\hline Formal education (none) & 113 & $16 \cdot 6$ & $191^{*}$ & $35 \cdot 2$ \\
\hline Literacy (unable) & 177 & $26 \cdot 1$ & $249^{*}$ & $45 \cdot 9$ \\
\hline Occupation (unemployed) & 110 & $16 \cdot 2$ & $192^{*}$ & $35 \cdot 4$ \\
\hline
\end{tabular}

IQR, interquartile range.

${ }^{*}$ Mean value was significantly different from that of the control group $(P \leq 0.05)$. 
The mean daily energy intake of the study population was $7791(\mathrm{sD} 2598) \mathrm{kJ} / \mathrm{d}(1862$ (sD 621) kcal/d). This did not differ between the controls $(7945$ (SD 2745) kJ/d (1899 $(\mathrm{SD} 656) \mathrm{kcal} / \mathrm{d})$ ) and cases $(7598(\mathrm{SD} 2385) \mathrm{kJ} / \mathrm{d}(1816$ (SD $570) \mathrm{kcal} / \mathrm{d}))(P=0.06)$. As for the macronutrients, the intake in the control group was higher for carbohydrates $(145 \mathrm{~g} /$ $4184 \mathrm{~kJ} v .136 \mathrm{~g} / 4184 \mathrm{~kJ}(145 \mathrm{~g} / 1000 \mathrm{kcal} v .136 \mathrm{~g} / 1000 \mathrm{kcal}) ;$ $P<0 \cdot 001)$ and lower for total fat $(34 \mathrm{~g} / 4184 \mathrm{~kJ} v .36 \mathrm{~g} / 4184 \mathrm{~kJ}$ $(34 \mathrm{~g} / 1000 \mathrm{kcal} v .36 \mathrm{~g} / 1000 \mathrm{kcal}) ; P<0.001)$ and dietary fibre $(16.0$ v. $17.5 \mathrm{~g} / \mathrm{d} ; \quad P=0.008)$ compared with the diabetes group. Protein intake was similar $(45 \mathrm{~g} / 4184 \mathrm{~kJ} \quad v .46 \mathrm{~g} /$ $4184 \mathrm{~kJ}(45 \mathrm{~g} / 1000 \mathrm{kcal} v .46 \mathrm{~g} / 1000 \mathrm{kcal}) ; P=0 \cdot 28)$.

\section{Dietary patterns}

By means of factor analysis, we derived dietary patterns in the total study population (Table 2). The first pattern explained $13.7 \%$ of the variance among food items and was named the 'purchase' dietary pattern. It was characterised by high intakes of sweets and sweet drinks, rice, foods rich in protein (red meat, poultry, eggs and milk), plant oils (vegetable oil and margarine), fruits and vegetables (carrot, lettuce and

Table 2. Rotated factor loadings for the two identified dietary patterns in the KDH (Kumasi Diabetes and Hypertension) Study*

\begin{tabular}{|c|c|c|}
\hline Food items & $\begin{array}{c}\text { 'Purchase' } \\
\text { dietary } \\
\text { pattern }\end{array}$ & $\begin{array}{c}\text { 'Traditional' } \\
\text { dietary } \\
\text { pattern }\end{array}$ \\
\hline Juice & 0.62 & -0.14 \\
\hline Sweets & 0.62 & -0.12 \\
\hline Rice & 0.58 & 0.04 \\
\hline Soft drinks & 0.58 & -0.06 \\
\hline Vegetable oil & 0.58 & -0.08 \\
\hline Milo (chocolate drink) & 0.54 & -0.06 \\
\hline Red meat & 0.50 & 0.02 \\
\hline Eggs & 0.48 & -0.03 \\
\hline Margarine & 0.46 & 0.09 \\
\hline Fruits & 0.46 & 0.38 \\
\hline Carrot & 0.43 & 0.30 \\
\hline Lettuce & 0.42 & 0.32 \\
\hline Milk & 0.40 & 0.06 \\
\hline Poultry & 0.40 & 0.19 \\
\hline Cucumber & 0.37 & 0.28 \\
\hline Plantain & -0.45 & 0.43 \\
\hline Green leafy vegetables & -0.02 & 0.54 \\
\hline Beans & 0.14 & 0.51 \\
\hline Garden egg & -0.16 & 0.49 \\
\hline Fish & -0.19 & 0.46 \\
\hline Maize (banku) & $0 \cdot 11$ & 0.43 \\
\hline Palm oil & 0.06 & 0.41 \\
\hline Okra & 0.20 & 0.35 \\
\hline Agushie (pumpkin seeds) & 0.20 & 0.33 \\
\hline Crab & 0.02 & 0.32 \\
\hline Bread & 0.20 & 0.29 \\
\hline Cassava & -0.20 & 0.28 \\
\hline Millet & -0.04 & 0.27 \\
\hline Yam & 0.06 & 0.21 \\
\hline Cocoyam & -0.02 & 0.20 \\
\hline Groundnut & 0.30 & 0.25 \\
\hline Porridge & 0.29 & 0.15 \\
\hline Coffee & 0.24 & 0.02 \\
\hline
\end{tabular}

${ }^{*}$ Factor loadings correspond to correlation coefficients between food intake and the dietary pattern score. cucumber), and low intake of plantain. Second, a 'traditional' dietary pattern explained a total variance of $8.5 \%$ and positively correlated with the intake of plantain, green leafy vegetables, beans, garden egg, fish, maize (banku), palm oil, okra and fruits. Further pattern solutions (3-5 factors) were examined, but did not reveal meaningful dietary patterns, i.e. the first pattern ('purchase') remained in all other solutions; two patterns were only characterised by a high intake of three (carrot, lettuce and cucumber) or two food items (milk and bread); and the 'traditional' dietary pattern separated into a 'starchy foods' pattern (characterised by high intakes of plantain, cassava, garden egg, fish and green leafy vegetables) and a 'beans and groundnut' pattern (characterised by high intakes of beans, groundnut, maize (banku) and millet).

The characteristics by quintiles of the 'purchase' dietary pattern among the 679 controls of the KDH (Kumasi Diabetes and Hypertension) Study are presented in Table 3, and those of the 'traditional' dietary pattern in Table 4. Participants in the highest quintile of the 'purchase' dietary pattern were younger, leaner and of better SES, and exhibited lower energy expenditure than those in the lower quintiles. In addition, the mean intakes in quintile 5 of the 'purchase' dietary pattern score were lower than those in the first up to the fourth quintile for sweets, eggs, margarine, vegetables, poultry and plantain ( 1.5 servings/week), moderate for sweet drinks, red meat, fruits and milk (3.5 servings/week) and high for rice ( $7 \cdot 0$ servings/week) (Table 3 ). As for the 'traditional' dietary pattern, participants in the highest quintile were characterised by higher age, increased measures of obesity, lower SES and higher energy expenditure when compared with those in the lower quintiles (Table 4). Also, in quintile 5 , the mean intakes were moderate for fruits, beans and okra (3.5 servings/week), and high for plantain, green leafy vegetables, garden egg, fish, banku and palm oil (5.5-7.0 servings/week).

\section{Dietary patterns and type 2 diabetes}

Table 5 shows the associations between dietary pattern scores and the risk of type 2 diabetes. The age- and sex-adjusted OR for type 2 diabetes of the highest quintile of the 'purchase' dietary pattern compared with the lowest quintile was $0 \cdot 10$ (95\% CI $0 \cdot 06,0 \cdot 17, P$ for trend $<0 \cdot 001$ ). This association was similar after further adjustment for family history of diabetes, unemployment status, educational attainment, literacy, smoking, daily energy expenditure, BMI and waist:hip ratio (models 2 and 3). In contrast, the OR in the highest quintile compared with that in the lowest quintile for the 'traditional' dietary pattern was 3.14 (95\% CI $2 \cdot 09,4.73, P$ for trend $<0 \cdot 001)$. This positive association slightly strengthened after further adjustments (models 2 and 3). Similarly to the quintile-based analysis, evaluating associations per $1 \mathrm{SD}$ increment of pattern scores revealed an inverse association for the 'purchase' dietary pattern (OR 0.41, 95\% CI 0.33, 0.50), whereas the 'traditional' dietary pattern was significantly associated with a higher odds for type 2 diabetes (OR 1.56, 95\% CI $1 \cdot 35,1 \cdot 81$ ) in the fully adjusted model. 
Table 3. Characteristics by quintiles of the 'purchase' dietary pattern among the 679 controls of the KDH (Kumasi Diabetes and Hypertension) Study (Mean values and standard deviations; number of participants and percentages; median values and interquartile ranges (IQR))

\begin{tabular}{|c|c|c|c|c|c|c|c|c|c|c|c|}
\hline \multirow[b]{3}{*}{ Characteristics } & \multicolumn{11}{|c|}{ 'Purchase' dietary pattern } \\
\hline & \multicolumn{2}{|c|}{ Quintile 1} & \multicolumn{2}{|c|}{ Quintile 2} & \multicolumn{2}{|c|}{ Quintile 3} & \multicolumn{2}{|c|}{ Quintile 4} & \multicolumn{2}{|c|}{ Quintile 5} & \multirow[b]{2}{*}{$P$ for trend ${ }^{*}$} \\
\hline & Mean & SD & Mean & SD & Mean & SD & Mean & SD & Mean & SD & \\
\hline$n$ & \multicolumn{2}{|c|}{135} & \multicolumn{2}{|c|}{136} & \multicolumn{2}{|c|}{136} & \multicolumn{2}{|c|}{136} & \multicolumn{2}{|c|}{136} & \\
\hline Age (years) & $58 \cdot 0$ & 12.5 & $52 \cdot 6$ & $13 \cdot 8$ & 47.5 & $13 \cdot 3$ & $43 \cdot 3$ & $13 \cdot 8$ & $32 \cdot 9$ & $12 \cdot 9$ & $<0.001$ \\
\hline $\mathrm{BMI}\left(\mathrm{kg} / \mathrm{m}^{2}\right)$ & $25 \cdot 7$ & $5 \cdot 7$ & $25 \cdot 8$ & 4.7 & 27 & $6 \cdot 1$ & $26 \cdot 4$ & 5 & $24 \cdot 1$ & 4.9 & 0.019 \\
\hline WHR & 0.88 & 0.08 & 0.88 & 0.06 & 0.87 & 0.07 & 0.86 & 0.07 & 0.82 & 0.07 & $<0.001$ \\
\hline \multirow{2}{*}{\multicolumn{12}{|c|}{$\begin{array}{l}\text { Energy expenditure } \\
\text { kcal/d }\end{array}$}} \\
\hline & & & & & & & & & & & \\
\hline Median & \multirow{2}{*}{\multicolumn{2}{|c|}{$\begin{array}{c}1477 \\
965-2027\end{array}$}} & \multirow{2}{*}{\multicolumn{2}{|c|}{$\begin{array}{c}1384 \\
962-1856\end{array}$}} & \multirow{2}{*}{\multicolumn{2}{|c|}{$\begin{array}{c}1423 \\
968-1931\end{array}$}} & \multirow{2}{*}{\multicolumn{2}{|c|}{$\begin{array}{c}1226 \\
981-1618\end{array}$}} & \multirow{2}{*}{\multicolumn{2}{|c|}{$\begin{array}{c}1211 \\
961-1645\end{array}$}} & 0.032 \\
\hline IQR & & & & & & & & & & & \\
\hline \multicolumn{12}{|l|}{$\mathrm{kJ} / \mathrm{d}$} \\
\hline & & & & & & & & & & & \\
\hline \multirow{2}{*}{ IQR } & 403 & 3481 & & 7766 & 405 & 3079 & & 770 & 402 & 8883 & \\
\hline & $n$ & $\%$ & $n$ & $\%$ & $n$ & $\%$ & $n$ & $\%$ & $n$ & $\%$ & \\
\hline Sex (female) & 106 & 78.5 & 107 & $78 \cdot 7$ & 103 & $75 \cdot 7$ & 118 & $86 \cdot 8$ & 89 & 65.4 & 0.001 \\
\hline Family history of diabetes (positive) & 38 & $28 \cdot 1$ & 29 & $21 \cdot 3$ & 33 & $24 \cdot 3$ & 32 & 23.5 & 39 & 28.7 & 0.584 \\
\hline Smoking (ever) & 8 & 5.9 & 6 & 4.4 & 5 & $3 \cdot 7$ & 3 & $2 \cdot 2$ & 7 & $5 \cdot 2$ & 0.609 \\
\hline Formal education (none) & 53 & $39 \cdot 3$ & 32 & 23.5 & 13 & $9 \cdot 6$ & 9 & $6 \cdot 6$ & 6 & 4.4 & $<0.001$ \\
\hline Literacy (unable) & 68 & $50 \cdot 4$ & 50 & $36 \cdot 8$ & 27 & 19.9 & 21 & $15 \cdot 4$ & 11 & $8 \cdot 1$ & $<0.001$ \\
\hline Occupation (unemployed) & 39 & 28.9 & 27 & $19 \cdot 9$ & 27 & $19 \cdot 9$ & 9 & $6 \cdot 6$ & 8 & 5.9 & $<0.001$ \\
\hline Frequency of food intake (servings/week) & Median & IQR & Median & IQR & Median & IQR & Median & IQR & Median & IQR & \\
\hline Juice & 0 & $0-0$ & 0 & $0-0.5$ & 0.5 & $0-1.5$ & 1 & $0-1.5$ & 3.5 & $1 \cdot 0-3.5$ & $<0.001$ \\
\hline Sweets & 0 & $0-0.5$ & 0 & $0-0.5$ & 0.5 & $0-0.5$ & 0.5 & $0.3-0.5$ & 1.5 & $0.5-1.5$ & $<0.001$ \\
\hline Rice & 1.5 & $0.5-3.5$ & 3.5 & $1.5-3.5$ & 4.5 & $3 \cdot 5-7 \cdot 0$ & 5.5 & $3.5-7.0$ & 7 & $5 \cdot 5-7 \cdot 0$ & $<0.001$ \\
\hline Soft drinks & 0.5 & $0-0.5$ & 0.5 & $0.5-1.5$ & 1.5 & $0.5-1.5$ & 1.5 & $0.5-3.5$ & 3.5 & $1.5-3.5$ & $<0.001$ \\
\hline Vegetable oil & 0.5 & $0.5-1.5$ & 1.5 & $1.5-3.5$ & 3.5 & $1.5-3.5$ & 3.5 & $1.5-5.5$ & 5.5 & $3.5-7.0$ & $<0.001$ \\
\hline Milo & 0.5 & $0-1.5$ & 1.5 & $0-1.5$ & 1.5 & $0.5-2.5$ & 1.5 & $0.5-3.5$ & 3.5 & $1.5-5.5$ & $<0.001$ \\
\hline Red meat & 0.5 & $0-1.5$ & 0.5 & $0.5-1.5$ & 1.5 & $0.5-3.5$ & 1.5 & $0.5-3.5$ & 3.5 & $1.5-7.0$ & $<0.001$ \\
\hline Eggs & 0.5 & $0-0.5$ & 0.5 & $0-0.5$ & 0.5 & $0.5-1.5$ & 1.5 & $0.5-1.5$ & 1.5 & $0.5-3.5$ & $<0.001$ \\
\hline Margarine & 0 & $0-0$ & 0 & $0-0.5$ & 0 & $0-0.5$ & 0.5 & $0-0.5$ & 1.5 & $0-3.5$ & $<0.001$ \\
\hline Fruits & 1.5 & $0.5-3.5$ & 1.5 & $1.5-3.5$ & 3.5 & $1.5-3.5$ & 3.5 & $1.5-3.5$ & 3.5 & $3.5-3.5$ & $<0.001$ \\
\hline Carrot & 0.5 & $0-0.5$ & 0.5 & $0-1.5$ & 0.5 & $0.5-1.5$ & 1.5 & $0.5-3.5$ & 1.5 & $0.5-3.5$ & $<0.001$ \\
\hline Lettuce & 0 & $0-0.5$ & 0.5 & $0-1.5$ & 0.5 & $0-1.5$ & 0.5 & $0.5-1.5$ & 1.5 & $0.5-3.5$ & $<0.001$ \\
\hline Milk & 0.5 & $0-0.5$ & 0.5 & $0-1.5$ & 0.5 & $0.5-3.5$ & 3.5 & $0.5-7.0$ & 3.5 & $1.5-7.0$ & $<0.001$ \\
\hline Poultry & 0.5 & $0.5-0.5$ & 0.5 & $0.5-1.5$ & 0.5 & $0.5-1.5$ & 0.5 & $0.5-1.5$ & 1.5 & $0.5-3.5$ & $<0.001$ \\
\hline Cucumber & 0 & $0-0$ & 0 & $0-0.5$ & 0.5 & $0-0.5$ & 0.5 & $0-1.5$ & 0.5 & $0-1.5$ & $<0.001$ \\
\hline Plantain & 7 & $3.5-7 \cdot 0$ & 3.5 & $1.5-7.0$ & 3.5 & $1.5-7.0$ & 3.5 & $1.5-5.5$ & 1.5 & $0.5-3.5$ & $<0.001$ \\
\hline Green leaves & 1.5 & $0.5-1.5$ & 3.5 & $1.5-4.5$ & 1.5 & $1.5-3.5$ & 3.5 & $1.5-5.5$ & 3.5 & $1.5-7.0$ & 0.012 \\
\hline Beans & 0.5 & $0.5-1.5$ & 1.5 & $0.5-3.5$ & 1.5 & $0.5-3.5$ & 1.5 & $0.5-3.5$ & 1.5 & $1.5-3.5$ & $<0.001$ \\
\hline Garden egg & 7 & $5 \cdot 5-7 \cdot 0$ & 7 & $3 \cdot 5-7 \cdot 0$ & 7 & $1.5-7.0$ & 7 & $1.5-7.0$ & 7 & $3 \cdot 5-7 \cdot 0$ & 0.007 \\
\hline Fish & 7 & $7 \cdot 0-7 \cdot 0$ & 7 & $6 \cdot 3-7 \cdot 0$ & 7 & $7 \cdot 0-7 \cdot 0$ & 7 & $7 \cdot 0-7 \cdot 0$ & 7 & $3 \cdot 5-7 \cdot 0$ & $<0.001$ \\
\hline Maize (banku) & 3.5 & $1.5-7.0$ & 3.5 & $1.5-7.0$ & 3.5 & $1.5-7.0$ & 3.5 & $1.5-7.0$ & 3.5 & $1.5-7.0$ & 0.010 \\
\hline Palm oil & 3.5 & $1.5-5.5$ & 3.5 & $1.5-3.5$ & 3.5 & $1.5-3.5$ & 3.5 & $1.5-5.5$ & 3.5 & $1 \cdot 5-6 \cdot 3$ & 0.005 \\
\hline Okra & 0.5 & $0-1.5$ & 1.5 & $0.5-3.5$ & 1.5 & $0.5-3.5$ & 1.5 & $0.5-3.5$ & 1.5 & $0.5-7.0$ & $<0.001$ \\
\hline
\end{tabular}

WHR, waist:hip ratio.

${ }^{*} P$ values for categorical variables were calculated by $\chi^{2}$ test. 
Table 4. Characteristics by quintiles of the 'traditional' dietary pattern among the 679 controls of the KDH (Kumasi Diabetes and Hypertension) Study

(Mean values and standard deviations; number of participants and percentages; median values and interquartile ranges (IQR))

\begin{tabular}{|c|c|c|c|c|c|c|c|c|c|c|c|}
\hline \multirow[b]{3}{*}{ Characteristics } & \multicolumn{11}{|c|}{ 'Traditional' dietary pattern } \\
\hline & \multicolumn{2}{|c|}{ Quintile 1} & \multicolumn{2}{|c|}{ Quintile 2} & \multicolumn{2}{|c|}{ Quintile 3} & \multicolumn{2}{|c|}{ Quintile 4} & \multicolumn{2}{|c|}{ Quintile 5} & \multirow[b]{2}{*}{$P$ for trend* } \\
\hline & Mean & SD & Mean & SD & Mean & SD & Mean & SD & Mean & SD & \\
\hline$n$ & \multicolumn{2}{|c|}{135} & \multicolumn{2}{|c|}{136} & \multicolumn{2}{|c|}{136} & \multicolumn{2}{|c|}{136} & \multicolumn{2}{|c|}{136} & \\
\hline Age (years) & 42 & $16 \cdot 5$ & 46 & $15 \cdot 8$ & $45 \cdot 3$ & 15 & $49 \cdot 1$ & $15 \cdot 6$ & 51.9 & $14 \cdot 3$ & $<0.001$ \\
\hline BMI $\left(\mathrm{kg} / \mathrm{m}^{2}\right)$ & 25.5 & 5.5 & $25 \cdot 2$ & $5 \cdot 2$ & $26 \cdot 1$ & $5 \cdot 3$ & $26 \cdot 3$ & 5.4 & $26 \cdot 1$ & 5.4 & $0 \cdot 169$ \\
\hline WHR & 0.84 & 0.08 & 0.86 & 0.09 & 0.86 & 0.07 & 0.86 & 0.07 & 0.88 & 0.06 & $<0.001$ \\
\hline \multirow{2}{*}{\multicolumn{12}{|c|}{$\begin{array}{l}\text { Energy expenditure } \\
\mathrm{kcal} / \mathrm{d}\end{array}$}} \\
\hline & & & & & & & & & & & \\
\hline Median & \multirow{2}{*}{\multicolumn{2}{|c|}{$\begin{array}{c}1231 \\
861-1720\end{array}$}} & \multirow{2}{*}{\multicolumn{2}{|c|}{$\begin{array}{c}1448 \\
1067-1883\end{array}$}} & \multirow{2}{*}{\multicolumn{2}{|c|}{$\begin{array}{c}1348 \\
1005-1800\end{array}$}} & \multirow{2}{*}{\multicolumn{2}{|c|}{$\begin{array}{c}1339 \\
966-1846\end{array}$}} & \multirow{2}{*}{\multicolumn{2}{|c|}{$\begin{array}{c}1408 \\
962-2009\end{array}$}} & 0.787 \\
\hline IQR & & & & & & & & & & & \\
\hline \multicolumn{12}{|l|}{$\mathrm{kJ} / \mathrm{d}$} \\
\hline \multirow{3}{*}{$\begin{array}{l}\text { Median } \\
\text { IQR }\end{array}$} & & & & & & & & & & & \\
\hline & 360 & 196 & & 878 & 420 & 531 & & 724 & 402 & 3406 & \\
\hline & $n$ & $\%$ & $n$ & $\%$ & $n$ & $\%$ & $n$ & $\%$ & $n$ & $\%$ & \\
\hline Sex (female) & 113 & 83.7 & 100 & 73.5 & 109 & $80 \cdot 2$ & 104 & $76 \cdot 5$ & 97 & 71.9 & $0 \cdot 107$ \\
\hline Family history of diabetes (positive) & 40 & $29 \cdot 6$ & 31 & $22 \cdot 8$ & 34 & 25 & 36 & $26 \cdot 5$ & 30 & $22 \cdot 2$ & 0.617 \\
\hline Smoking (ever) & 2 & 1.5 & 6 & 4.4 & 8 & $5 \cdot 9$ & 5 & 3.7 & 8 & 5.9 & 0.352 \\
\hline Formal education (none) & 15 & $11 \cdot 1$ & 24 & $17 \cdot 7$ & 24 & $17 \cdot 7$ & 26 & $19 \cdot 1$ & 24 & $17 \cdot 8$ & 0.423 \\
\hline Literacy (unable) & 24 & $17 \cdot 8$ & 39 & 28.7 & 34 & 25 & 43 & $31 \cdot 6$ & 37 & $27 \cdot 4$ & 0.106 \\
\hline Occupation (unemployed) & 15 & $11 \cdot 1$ & 19 & 14 & 20 & $14 \cdot 7$ & 27 & $19 \cdot 9$ & 29 & 21.5 & 0.123 \\
\hline Frequency of food intake (servings/week) & Median & IQR & Median & IQR & Median & IQR & Median & IQR & Median & IQR & \\
\hline Juice & 0.5 & $0-1.5$ & 0 & $0-1.5$ & 0.5 & $0-1.5$ & 0.5 & $0-1.5$ & 0.5 & $0-1.5$ & 0.078 \\
\hline Sweets & 0.5 & $0-0.5$ & 0.5 & $0-0.5$ & 0.5 & $0-0.5$ & 0.5 & $0-0.5$ & 0.5 & $0-0.5$ & 0.079 \\
\hline Rice & 3.5 & $1.5-7.0$ & 3.5 & $1.5-6.3$ & 3.5 & $1.5-7.0$ & 3.5 & $1.5-7.0$ & 5.5 & $3.5-7.0$ & 0.019 \\
\hline Soft drinks & 1.5 & $0.5-1.5$ & 0.5 & $0.5-1.5$ & 1.5 & $0.5-3.5$ & 0.5 & $0.5-1.5$ & 1.5 & $0.5-3.5$ & 0.480 \\
\hline Vegetable oil & 3.5 & $1.5-5.5$ & 3.5 & $1.5-3.5$ & 3.5 & $1.5-3.5$ & 1.5 & $0.5-3.5$ & 3.5 & $1.5-3.5$ & 0.011 \\
\hline Milo & 1.5 & $0.5-3.5$ & 1.5 & $0-3.5$ & 1.5 & $0.5-3.5$ & 1.5 & $0.5-3.5$ & 1.5 & $0.5-3.5$ & 0.400 \\
\hline Red meat & 1.5 & $0.5-3.5$ & 1.5 & $0.5-3.5$ & 1.5 & $0.5-3.5$ & 1.5 & $0.5-3.5$ & 1.5 & $0.5-3.5$ & 0.831 \\
\hline Eggs & 0.5 & $0.5-1.5$ & 0.5 & $0.5-1.5$ & 0.5 & $0.5-1.5$ & 0.5 & $0.5-1.5$ & 0.5 & $0.5-1.5$ & 0.996 \\
\hline Margarine & 0 & $0-0.5$ & 0 & $0-0.5$ & 0 & $0-0.5$ & 0 & $0-0.5$ & 0.5 & $0-1.5$ & 0.001 \\
\hline Fruits & 1.5 & $1.5-3.5$ & 1.5 & $1.5-3.5$ & 3.5 & $1.5-3.5$ & 3.5 & $1.5-3.5$ & 3.5 & $3.5-3.5$ & $<0.001$ \\
\hline Carrot & 0.5 & $0-1.5$ & 0.5 & $0-1.5$ & 1.5 & $0.5-1.5$ & 0.5 & $0.3-1.5$ & 1.5 & $0.5-3.5$ & $<0.001$ \\
\hline Lettuce & 0.5 & $0-0.5$ & 0.5 & $0-1.5$ & 0.5 & $0-1.5$ & 0.5 & $0-1.5$ & 1.5 & $0.5-1.5$ & $<0.001$ \\
\hline Milk & 1.5 & $0.5-3.5$ & 1 & $0.5-3.5$ & 1.5 & $0.5-3.5$ & 0.5 & $0.5-3.5$ & 1.5 & $0.5-5.5$ & 0.577 \\
\hline Poultry & 0.5 & $0.5-1.5$ & 0.5 & $0.5-1.5$ & 0.5 & $0.5-1.5$ & 0.5 & $0.5-1.5$ & 0.5 & $0.5-1.5$ & 0.001 \\
\hline Cucumber & 0.5 & $0-0.5$ & 0 & $0-0.5$ & 0 & $0-0.5$ & 0 & $0-0.5$ & 0.5 & $0-1.5$ & $<0.001$ \\
\hline Plantain & 1.5 & $0.5-3.5$ & 3.5 & $1.5-5.5$ & 3.5 & $1.5-7.0$ & 3.5 & $3.5-7.0$ & 7 & $3.5-7.0$ & $<0.001$ \\
\hline Green leaves & 1.5 & $0.5-1.5$ & 1.5 & $1.5-3.5$ & 1.5 & $0.5-3.5$ & 3.5 & $1.5-5.5$ & 7 & $3.5-7.0$ & $<0.001$ \\
\hline Beans & 1.5 & $0.5-1.5$ & 1.5 & $0.5-3.5$ & 1.5 & $0.5-3.5$ & 1.5 & $0.5-3.5$ & 3.5 & $1.5-7.0$ & $<0.001$ \\
\hline Garden egg & 1.5 & $1.5-3.5$ & 3.5 & $3.5-7.0$ & 7 & $3.5-7.0$ & 7 & $7 \cdot 0-7 \cdot 0$ & 7 & $7 \cdot 0-7.0$ & $<0.001$ \\
\hline Fish & 3.5 & $3.5-7.0$ & 7 & $4.5-7.0$ & 7 & $7 \cdot 0-7 \cdot 0$ & 7 & $7 \cdot 0-7 \cdot 0$ & 7 & $7 \cdot 0-7 \cdot 0$ & $<0.001$ \\
\hline Maize (banku) & 1.5 & $1.5-3.5$ & 1.5 & $1.5-3.5$ & 3.5 & $1.5-7.0$ & 3.5 & $1.5-7.0$ & 7 & $3.5-7.0$ & $<0.001$ \\
\hline Palm oil & 1.5 & $1.5-3.5$ & 3.5 & $1.5-3.5$ & 3.5 & $1.5-3.5$ & 3.5 & $3 \cdot 5-6 \cdot 3$ & 5.5 & $3.5-7.0$ & $<0.001$ \\
\hline Okra & 0.5 & $0.5-1.5$ & 0.5 & $0.5-1.5$ & 1.5 & $0.5-3.5$ & 1.5 & $0.5-3.5$ & 3.5 & $1.5-7.0$ & $<0.001$ \\
\hline
\end{tabular}




\section{Discussion}

We identified and characterised dietary patterns and examined their associations with type 2 diabetes. Given the limited number of studies from SSA, our findings contribute uniquely to the literature. There were two dietary patterns in this urban Ghanaian population. The 'purchase' dietary pattern was characterised by a high consumption of sweets, rice, protein-rich foods (red meat, poultry, eggs and milk), fruits and vegetables and low consumption of plantain; this was inversely associated with the risk of type 2 diabetes. The 'traditional' dietary pattern was characterised by a high intake of plantain, green leafy vegetables, beans, garden egg, fruits, fish, fermented maize products and palm oil; this was associated with an increased odds for type 2 diabetes.

\section{Dietary patterns in sub-Saharan Africa}

With respect to SSA, only five cross-sectional studies in comparatively small and specific populations have attempted to identify dietary patterns by some sort of factor analysis or cluster analysis: one cross-sectional study among the Cameroon defence forces ( $n$ 571) identified two dietary patterns by means of factor analysis ${ }^{(25)}$. The 'fruit and vegetable' pattern was similar to our mixed 'purchase' dietary pattern with high factor loadings for fruits, green and dark yellow vegetables, tubers, oils and fats, fish and seafood, rice, milk and milk products, pasta, soft drinks, cakes and cookies, sweets and meat (poultry, red meat, organ meat and bush meat). In contrast, the 'meat' pattern exhibited high factor loadings for bush meat, poultry, and red meat, but low factor loadings for sweets, cakes and sugar. Among 1086 elderly in Botswana, five dietary patterns were revealed using factor analysis ${ }^{(26)}$ : 'beer', 'meat and fruit', 'vegetable and bread', 'seasonal produce', and 'milk, tea and candy' patterns, whereby only the 'meat and fruit' pattern exhibited some similarities to our 'purchase' dietary pattern. Furthermore, among 1072 urban Burkinans, a 'snacking' pattern (fried foods, sugar-sweetened products, cereals and dairy products) and a 'modern foods' dietary pattern (processed meats, eggs, low in nuts, seeds, cereals and beans) were identified by principal component analysis ${ }^{(27)}$. However, these did not show similarities with our dietary patterns. Keding et $a{ }^{(28)}$ observed five dietary patterns in rural Tanzania ( $n$ 252) by applying principal component analysis: (1) 'purchase' dietary pattern (characterised by bread and cakes, sugar, and black tea); (2) 'traditional-coast' (characterised by fruits, nuts, starchy plants and fish); (3) 'traditional-inland' (characterised by cereals, oils and fats, and vegetables); (4) 'pulses' (characterised mainly by pulses, with few or no vegetables); (5) 'animal products' (characterised by a high consumption of meat, eggs and/or milk) ${ }^{(28)}$. The best comparable dietary patterns were identified among 200 urban Beninese residents by cluster analysis ${ }^{(29)}$ : a 'traditional' dietary pattern (characterised by high intakes of grains, fruits, fish and green leafy vegetables) and a 'transitional' one (characterised by high intakes of bread, pasta, roots, nuts, meat, eggs, dairy, fats and sweets). As a mixed pattern, the latter unites 
imported and traditional foods and is similar to our 'purchase' dietary pattern.

These data highlight the difficulties to establish dietary patterns in African populations and to compare, not to say transfer, established patterns with other SSA regions.

\section{Characteristics of dietary patterns and diet-disease associations in sub-Saharan Africa}

It is not trivial to find common characteristics of the identified dietary patterns in SSA. For example, the pattern with a high consumption of vegetables in Benin predominated in individuals of low SES ${ }^{(29)}$, whereas in Botswana, such pattern prevailed in households with children ${ }^{(26)}$. With respect to a pattern loading high on meat intake, this was characterised by low educational level in Cameroon ${ }^{(25)}$, while in Benin, participants of high SES adhered to such pattern ${ }^{(29)}$. In part, this is in line with the observations of the present study. Adherence to dietary patterns was associated with SES. Participants with a high score of the 'purchase' dietary pattern were characterised by a better SES, whereas those with a high score of the 'traditional' dietary pattern were more deprived. Indeed, the 'purchase' dietary pattern included food items (sweets, red meat and poultry) that are expensive for the majority of the population in this area. It seems that particularly people above the average income level and with better knowledge of healthy food adhere to this. In contrast, low income and poor education might favour the adherence to the 'traditional' dietary pattern. Similarly, in the aforementioned study from Benin, the proxy indicator for SES 'birthplace' was associated with the 'transitional' (urban) and 'traditional' dietary patterns (rural) ${ }^{(29)}$.

As for Ghana, the proportion of the population living in urban areas has increased from 23 to $51 \%$ during the past 50 years, with the second highest proportion of urban citizens $(61 \%)$ in the Ashanti Region ${ }^{(30)}$. In Benin, no clear associations of dietary patterns (characterised by urban $v$. rural citizenship and high $v$. low SES), with self-reported health status, were observed ${ }^{(29)}$. Nevertheless, urbanisation and the epidemic of type 2 diabetes are paralleling in $\operatorname{SSA}^{(15,31)}$. Thus, it is justified to hypothesise that peasants from rural Ghana moved to the cities, where they faced lower income and altered food availability. In this situation, well-known traditional foods that are satiating and inexpensive, such as carbohydrate-dense foods of the 'traditional' dietary pattern, appear preferable. This could be a possible explanation for our observed positive association between the 'traditional' dietary pattern and type 2 diabetes. Unfortunately, we have no information about movements from rural to urban areas and therefore cannot investigate this hypothesis so far. Of note, such associations between SES and the adherence to dietary patterns have been observed in Western populations, too. So-called prudent or healthier patterns were associated with increased income, better education and older age ${ }^{(32)}$

With respect to diet-disease relationships in the African region, the scarce data remain inconclusive. The Cameroon study was designed to identify patterns associated with hypertension and revealed that the fourth quartile of the 'fruit and vegetable' pattern compared with the first quartile reduced the risk of hypertension by $60 \%{ }^{(25)}$. The Botswanian study by Maruapula \& Chapman-Novakofski ${ }^{(26)}$ did not cover analyses on dietary patterns and health outcomes. In Burkina Faso, obesity was the outcome of interest. There was no relationship with the 'modern foods' pattern; however, there was a positive association with the 'snacking' pattern ${ }^{(27)}$. Similarly, in Tanzania, the 'purchase' dietary pattern showed the strongest positive association with $\mathrm{BMI}^{(28)}$. This contrasts the lack of association in Benin ${ }^{(29)}$.

Clearly, these findings warrant further investigations to understand the determinants of adherence to dietary patterns in SSA.

\section{Associations of dietary patterns with type 2 diabetes in other populations}

To our knowledge, the present study is the first to investigate the associations between dietary patterns and the risk of type 2 diabetes in SSA. The inverse association of the 'purchase' dietary pattern, which was rich in fruits and vegetables, appears to be consistent with previous studies reporting an inverse association for patterns sometimes called 'prudent' or 'healthy' (characterised by higher intakes of fruits and vegetables) ${ }^{(33,34)}$. However, there are important differences in pattern structure observed in the present study compared with patterns evaluated in European ${ }^{(33,35)}$ US $^{(34,36)}$ or Asian populations ${ }^{(10,11,37)}$. The 'purchase' dietary pattern was also characterised by a high intake of red meat. Previously, this has not been labelled 'prudent', but rather 'Western', and was associated with increased risk in other populations ${ }^{(34,36,37)}$. This is also true for the consumption of sweets in such 'Western' patterns, which is positively associated with type 2 diabetes in Western populations ${ }^{(13,36,39)}$, but inversely in the present study. While the consumption of sweets was low in our study population and the types of red meat differed from those in Western populations which might explain that the pattern was - in contrast to so-called 'Western' patterns - inversely associated with the risk of type 2 diabetes, the present study highlights that dietary patterns derived by exploratory methods are specific for this African study population. Nevertheless, exploration of food variability in the KDH Study was highly dependent on the adequacy of the FFQ. A local specificity might be postulated and even more pronounced for the 'traditional' dietary pattern, which consisted of fermented maize products, palm oil and other traditional foods which are merely absent in diets in Western and Asian regions. Clearly, the observed relationships between dietary patterns and type 2 diabetes require verification in other regions of West Africa.

The present study holds several strengths and some limitations. The characterisation of the nutritional behaviour and identification of dietary patterns in a Ghanaian population is unique. Also, compared with other studies in SSA ${ }^{(25,28,29)}$, the sample size of the present study is large. In the context of scarce epidemiological data, a case-control design is useful to establish hypotheses on the association between dietary patterns and the risk of type 2 diabetes. However, 
prospective studies may provide stronger evidence for the causal relationship between dietary patterns and the risk of diabetes. Thus, we cannot exclude that reverse causation explains our observations. Particularly, most of the diabetes cases in the present study (97\%) have already been known to the Komfo Anokye Teaching Hospital, and were under regular monitoring. However, routine diabetes management does not comprise nutritional counselling. Consequently, we do not expect that awareness of type 2 diabetes is accompanied by a change in nutritional behaviour. Nevertheless, we cannot exclude such reverse causation.

By means of culturally sensitive $24 \mathrm{HDR}$ and FFQ, the present study provides qualitative and quantitative information of food intake. We are aware that both methods exhibit their limitations. While $24 \mathrm{HDR}$ are useful to describe the short-term and current diet in surveys, their performance is limited in case-control settings to reproduce a retrospective exposition. However, the classic weaknesses of $24 \mathrm{HDR}$, i.e. forgotten foods (recall bias) and non-naming of foods (under-reporting), seem less problematic in SSA than in Europe or North America ${ }^{(40)}$. Specifically, under-reporting might have occurred in participants with diabetes and/or overweight, who may have tended to give socially desirable answers. However, in this region, obesity is perceived as a marker of affluence ${ }^{(41)}$ and is linked to health and beauty in Ghanaian women ${ }^{(16)}$. The majority of the Ghanaian women $(72 \%)$ are not satisfied with their body size, and $30 \%$ of them would prefer a heavier body size. In addition, $61 \%$ of the women who are satisfied with their body size are overweight or obese ${ }^{(42)}$. Furthermore, the determinants of food choice are less influenced by social desirability than more by convenience, availability and price ${ }^{(43)}$. Indeed, $24 \mathrm{HDR}$ perform well in regions with high rates of illiteracy and of low SES, particularly when applied by interviewers of the same cultural background speaking the local language. Clearly, the inter- and intra-individual variance of a $24 \mathrm{HDR}$ limits information on the actual, individual diet. However, 24HDR are useful to compare energy and nutrient intakes between population groups ${ }^{(44)}$, specifically when applying local household measures and food composition tables. In contrast to $24 \mathrm{HDR}, \mathrm{FFQ}$ depict the long-term usual diet. As with all retrospective assessment methods, FFQ run the risk of recall bias and under/overestimation of portion sizes; their quantitative precision may be limited. Nevertheless, they are substantially cheaper than $24 \mathrm{HDR}^{(44)}$ and feasible to measure nutrition exposition in case-control or cross-sectional studies. If the food list is culturally sensitive as in the present study, FFQ exhibit excellent properties to assess nutritional behaviour in this study setting.

In the present study population of low SES, both dietary patterns were strongly associated with proxy markers of SES (education, literacy and unemployment). Surprisingly, the strength of associations only slightly attenuated after adjusting for these. Still, we cannot rule out that SES is imperfectly measured in the present study, and that residual confounding by SES may partly explain our observed associations.

\section{Conclusion}

In conclusion, we identified two main dietary patterns in an urban Ghanaian population, which differed from previous reports among Western and Asian populations. Our findings suggest that a 'purchase' dietary pattern (characterised by high intakes of sweets, rice, meat, fruits and vegetables) and a 'traditional' dietary pattern (characterised by high intakes of fruits, plantain, green leafy vegetables, fish, fermented maize products and palm oil) are associated with the risk of type 2 diabetes in this population. Clearly, the evaluation and investigation of determinants of adherence to dietary patterns warrants further investigations in SSA.

\section{Supplementary material}

To view supplementary material for this article, please visit http://dx.doi.org/10.1017/S000711451400052X

\section{Acknowledgements}

The authors thank all participants at Komfo Anokye Teaching Hospital and acknowledge the study team of the KDH Study for on-site recruitment, data and sample collection as well as laboratory analysis.

The present study was supported by Charité - Universitaetsmedizin Berlin (grant no. 89539150). The funder had no role in the design, analysis or writing of this article.

The authors' contributions are as follows: L. K. F. performed the statistical analysis, data interpretation and wrote the manuscript; I. D., J. K. and M. B. S. supervised the study conduct, provided statistical expertise and revised the manuscript; I. D., G. B.-A. and F. P. M. conceived and designed the study and were responsible for recruitment, interviews and examinations of the study participants.

None of the authors has any conflicts of interest.

\section{References}

1. Trichopoulos D \& Lagiou P (2001) Dietary patterns and mortality. Br J Nutr 85, 133-134.

2. Hu FB (2002) Dietary pattern analysis: a new direction in nutritional epidemiology. Curr Opin Lipidol 13, 3-9.

3. Schulze MB \& Hoffmann K (2006) Methodological approaches to study dietary patterns in relation to risk of coronary heart disease and stroke. Br J Nutr 95, 860-869.

4. Schulze MB \& Hu FB (2002) Dietary patterns and risk of hypertension, type 2 diabetes mellitus, and coronary heart disease. Curr Atheroscler Rep 4, 462-467.

5. Kennedy ET, Ohls J, Carlson S, et al. (1995) The Healthy Eating Index: design and applications. J Am Diet Assoc 95, 1103-1108.

6. Kim S, Haines PS, Siega-Riz AM, et al. (2003) The Diet Quality Index-International (DQI-I) provides an effective tool for crossnational comparison of diet quality as illustrated by China and the United States. J Nutr 133, 3476-3484.

7. Newby PK \& Tucker KL (2004) Empirically derived eating patterns using factor or cluster analysis: a review. Nutr Rev 62, 177-203.

8. Hoffmann K, Schulze MB, Schienkiewitz A, et al. (2004) Application of a new statistical method to derive dietary 
patterns in nutritional epidemiology. Am J Epidemiol 159, 935-944.

9. Esposito K, Kastorini CM, Panagiotakos DB, et al. (2010) Prevention of type 2 diabetes by dietary patterns: a systematic review of prospective studies and meta-analysis. Metab Syndr Relat Disord 8, 471-476.

10. Yu R, Woo J, Chan R, et al. (2011) Relationship between dietary intake and the development of type 2 diabetes in a Chinese population: the Hong Kong Dietary Survey. Public Health Nutr 14, 1133-1141.

11. Odegaard AO, Koh WP, Butler LM, et al. (2011) Dietary patterns and incident type 2 diabetes in Chinese men and women: the Singapore Chinese Health Study. Diabetes Care 34, 880-885.

12. Bauer F, Beulens JW, van der AD, et al. (2013) Dietary patterns and the risk of type 2 diabetes in overweight and obese individuals. Eur J Nutr 52, 1127-1134.

13. Malik VS, Fung TT, van Dam RM, et al. (2012) Dietary patterns during adolescence and risk of type 2 diabetes in middle-aged women. Diabetes Care 35, 12-18.

14. Erber E, Hopping BN, Grandinetti A, et al. (2010) Dietary patterns and risk for diabetes: the multiethnic cohort. Diabetes Care 33, 532-538.

15. Amoah AG, Owusu SK \& Adjei S (2002) Diabetes in Ghana: a community based prevalence study in Greater Accra. Diabetes Res Clin Pract 56, 197-205.

16. Amoah AG (2003) Sociodemographic variations in obesity among Ghanaian adults. Public Health Nutr 6, 751-757.

17. Danquah I, Bedu-Addo G, Terpe KJ, et al. (2012) Diabetes mellitus type 2 in urban Ghana: characteristics and associated factors. BMC Public Health 12, 210.

18. Frank LK, Heraclides A, Danquah I, et al. (2013) Measures of general and central obesity and risk of type 2 diabetes in a Ghanaian population. Trop Med Int Health 18, 141-151.

19. World Health Organization (1999) Definition, Diagnosis and Classification of Diabetes Mellitus and its Complication: Report of a WHO Consultation. Part 1: Diagnosis and Classification of Diabetes Mellitus. Geneva: WHO. http:// whqlibdoc.who.int/hq/1999/WHO_NCD_NCS_99.2.pdf

20. Ghana Statistical Service. (GSS), Ghana Health Service (GHS) \& ICF Macro (2009) Ghana Demographic and Health Survey 2008. Accra: GSS, GHS, and ICF Macro. http://www.measuredhs. com/pubs/pdf/FR221/FR221\%5B13Aug2012\%5D.pdf

21. Conway JM, Ingwersen LA, Vinyard BT, et al. (2003) Effectiveness of the US Department of Agriculture 5-step multiple-pass method in assessing food intake in obese and non-obese women. Am J Clin Nutr 77, 1171-1178.

22. Ainsworth BE, Haskell WL, Leon AS, et al. (1993) Compendium of physical activities: classification of energy costs of human physical activities. Med Sci Sports Exerc 25, 71-80.

23. Food Research Institute (C.S.I.R) (1975) Measurements of Foods Commonly used in Ghana. Rome: Food and Agricultural Organization of the United Nations.

24. Food Research Institute (C.S.I.R) (1975) Composition of Foods Commonly Used in Ghana. Rome: Food and Agricultural Organization of the United Nations.

25. Nkondjock A \& Bizome E (2010) Dietary patterns associated with hypertension prevalence in the Cameroon defence forces. Eur J Clin Nutr 64, 1014-1021.

26. Maruapula S \& Chapman-Novakofski K (2007) Health and dietary patterns of the elderly in Botswana. J Nutr Educ Behav 39, 311-319.
27. Becquey E, Savy M, Danel P, et al. (2010) Dietary patterns of adults living in Ouagadougou and their association with overweight. Nutr J 9, 13.

28. Keding GB, Msuya JM, Maass BL, et al. (2011) Dietary patterns and nutritional health of women: the nutrition transition in rural Tanzania. Food Nutr Bull 32, 218-226.

29. Sodjinou R, Agueh V, Fayomi B, et al. (2009) Dietary patterns of urban adults in Benin: relationship with overall diet quality and socio-demographic characteristics. Eur J Clin Nutr 63, 222-228.

30. Ghana Statistical Service (2010) Population and Housing Census Summary Results of Final Report. Ghana: GSS. http://www.statsghana.gov.gh/docfiles/2010phc/Census 2010_Summary_report_of_final_results.pdf

31. Abubakari AR \& Bhopal RS (2008) Systematic review on the prevalence of diabetes, overweight/obesity and physical inactivity in Ghanaians and Nigerians. Public Health 122, 173-182.

32. Kant AK (2004) Dietary patterns and health outcomes. J Am Diet Assoc 104, 615-635.

33. Montonen J, Knekt P, Harkanen T, et al. (2005) Dietary patterns and the incidence of type 2 diabetes. $A m J$ Epidemiol 161, 219-227.

34. van Dam RM, Rimm EB, Willett WC, et al. (2002) Dietary patterns and risk for type 2 diabetes mellitus in U.S. men. Ann Intern Med 136, 201-209.

35. Heidemann C, Hoffmann K, Spranger J, et al. (2005) A dietary pattern protective against type 2 diabetes in the European Prospective Investigation into Cancer and Nutrition (EPIC)-Potsdam Study cohort. Diabetologia $\mathbf{4 8}$, 1126-1134.

36. Fung TT, Schulze M, Manson JE, et al. (2004) Dietary patterns, meat intake, and the risk of type 2 diabetes in women. Arch Intern Med 164, 2235-2240.

37. Villegas R, Yang G, Gao YT, et al. (2010) Dietary patterns are associated with lower incidence of type 2 diabetes in middle-aged women: the Shanghai Women's Health Study. Int J Epidemiol 39, 889-899.

38. van Dam RM, Willett WC, Rimm EB, et al. (2002) Dietary fat and meat intake in relation to risk of type 2 diabetes in men. Diabetes Care 25, 417-424.

39. Hodge AM, English DR, O'Dea K, et al. (2007) Dietary patterns and diabetes incidence in the Melbourne Collaborative Cohort Study. Am J Epidemiol 165, 603-610.

40. Harrison GG, Galal OM, Ibrahim M, et al. (2000) Underreporting of food intake by dietary recall is not universal: a comparison of data from Egyptian and American women. J Nutr 130, 2049-2054.

41. Mbanya JC, Motala AA, Sobngwi E, et al. (2010) Diabetes in sub-Saharan Africa. Lancet 375, 2254-2266.

42. Benkeser RM, Biritwum R \& Hill AG (2012) Prevalence of overweight and obesity and perception of healthy and desirable body size in urban, Ghanaian women. Ghana Med J 46, 66-75

43. Lopriore C \& Muehlhoff E (2003) Food Security and Nutrition Trends in West Africa - Challenges and the Way Forward; Nutrition Programmes Service, Food and Agriculture Organization. Rome: FAO. ftp://ftp.fao.org/es/ esn/nutrition/ouagafinal.pdf

44. Subar AF, Kipnis V, Troiano RP, et al. (2003) Using intake biomarkers to evaluate the extent of dietary misreporting in a large sample of adults: the OPEN study. Am J Epidemiol 158, 1-13. 